\title{
Context-Dependent Neuronal Activity in the Lateral Amygdala Represents Fear Memories after Extinction
}

\author{
Jennifer A. Hobin, ${ }^{1}$ Ki A. Goosens, ${ }^{1}$ and Stephen Maren ${ }^{1,2}$ \\ ${ }^{1}$ Department of Psychology and ${ }^{2}$ Neuroscience Program, University of Michigan, Ann Arbor, Michigan 48109-1109
}

The context in which fear memories are extinguished has important implications for treating human fear and anxiety disorders. Extinction of Pavlovian fear conditioning is context specific; after extinction, fear responses are reduced only in the extinction context and remain elevated in every other context. Contextual modulation of spike firing in the amygdala is a putative mechanism for the contextspecific expression of extinguished fear. To test this possibility, we conditioned rats to fear two auditory conditional stimuli (CSs) and then extinguished each CS in separate and distinct contexts. Thereafter, single-unit activity in the lateral nucleus of the amygdala (LA) and freezing behavior were recorded during tests in which each CS was presented in each extinction context. Hence, each CS was tested in its own extinction context and in the context of the other CS. Conditional freezing was context dependent; fear to an extinguished CS was low in its own extinction context and high in the other test context. Similarly, the majority of LA neurons exhibited context-dependent spike firing; short-latency spike firing was greater to both CSs when they were presented outside of their own extinction context. In contrast, behavioral and neuronal responses to either non-extinguished CSs or habituated auditory stimuli were not contextually modulated. Context-dependent neuronal activity in the LA may be an important mechanism for disambiguating the meaning of fear signals, thereby enabling appropriate behavioral responses to such stimuli.

Key words: Pavlovian fear conditioning; extinction; lateral amygdala; context; retrieval; single-unit; rats

\section{Introduction}

The conditions under which fear memories are extinguished have important implications for treating human fear and anxiety disorders (Bouton et al., 2001). Contextual stimuli are particularly important in regulating the expression of fear responses after these responses have been extinguished. Specifically, these responses are attenuated if fear-eliciting stimuli are presented in the extinction context but renewed if these stimuli are presented outside the extinction context. Although the contextual modulation of emotional responses has been widely studied (for review, see Bouton, 1993), the neural mechanisms enabling this modulation are not well characterized. Here we examine the neural mechanisms underlying the context specificity of Pavlovian fear conditioning in rats, a form of aversive conditioning that models clinical fear and anxiety disorders in humans.

In Pavlovian fear conditioning, a neutral conditional stimulus (CS; a tone) is paired with an aversive unconditional stimulus (US; a footshock). After pairing, presentation of the CS alone elicits a constellation of conditional fear responses (CRs). These

Received April 24, 2003; revised July 18, 2003; accepted July 22, 2003.

The research was supported by National Institute of Mental Health Grants R29MH57865 and R01MH65961 (S.M.) and a Department of Defense National Defense Science and Engineering Graduate fellowship (J.A.H.). K.A.G. was a Howard Hughes predoctoral fellow at the time this work was completed. We thank Kevin A. Corcoran for technical assistance and Drs. Kent C. Berridge, Terry E. Robinson, and Edward E. Smith for comments on previous versions of this manuscript.

Correspondence should be addressed to Stephen Maren, Department of Psychology, University of Michigan, 525 East University Avenue, Ann Arbor, MI 48109-1109. E-mail: maren@umich.edu.

K. A. Goosens's present address: Department of Biological Sciences, Stanford University, Stanford, CA 940255020.

Copyright $\odot 2003$ Society for Neuroscience $\quad$ 0270-6474/03/238410-07\$15.00/0
CRs are not context specific; presenting the CS in any context will elicit CRs. Once extinguished, however, memory for the CS becomes context specific (Bouton and Bolles, 1979; Harris et al., 2000; Corcoran and Maren, 2001); that is, the decrement in the CR that occurs during extinction training is only evident when animals are tested in the extinction context. After extinction, the memory for the CS is ambiguous (it predicts shock in one phase of training but not in another), and context resolves this ambiguity (Bouton, 1993; Maren and Holt, 2000).

A number of studies reveal that neuronal responses (e.g., spike firing) to sensory (Zipser et al., 1996; Das and Gilbert, 1999; Rossi et al., 2001; Albright and Stoner, 2002; Herzog and Fahle, 2002; Malone et al., 2002) and emotional (Diamond and Weinberger, 1989; Freeman et al., 1997) stimuli may vary greatly depending on the circumstances, or context, surrounding their presentation. This neural adaptation greatly increases information storage capacity and enables behavior to adapt to changing environmental demands.

Neurons encoding fear memories are located in the lateral nucleus of the amygdala (LA) (Davis, 1992; LeDoux, 2000; Maren, 2001), and LA neurons exhibit changes in spike firing during the acquisition and extinction of conditional fear (Quirk et al., 1995, 1997; Maren, 2000; Paré and Collins, 2000). Insofar as LA neurons mediate fear memory expression and exhibit context dependence in some emotional learning tasks (Freeman et al., 1997), we hypothesized that CS-elicited firing of LA neurons would be contextually modulated after extinction training; that is, when presented with an ambiguous CS, neuronal activity in the LA would reflect the encoding of contextual information enabling animals to select an appropriate, context-specific fear re- 
sponse. To test this idea, we trained rats using a fear extinction procedure that yields context-specific memories for two different CSs. We then recorded single-unit activity in the LA during presentations of the extinguished CSs either within or outside of their extinction contexts.

\section{Materials and Methods}

Subjects and surgery. We used male Long-Evans rats provided by a commercial supplier (Harlan Sprague Dawley, Indianapolis, IN) weighing between 300 and $500 \mathrm{gm}$. After arrival, rats were housed singly in hanging plastic cages. Animals were kept on a 14/10 hr light/dark cycle (lights on at 7:00 A.M.) and provided with food and water ad libitum. At least $1 \mathrm{~d}$ after extinction, rats under sodium pentobarbital anesthesia $(65 \mathrm{mg} / \mathrm{kg}$, i.p.) were stereotaxically implanted with a multi-wire recording electrode assembly aimed at the right dorsal LA (LAd; $3.3 \mathrm{~mm}$ posterior to bregma; $5.5 \mathrm{~mm}$ lateral to bregma; $6.0-6.4 \mathrm{~mm}$ ventral to dura). Electrodes consisted of eight $25 \mu \mathrm{m}$ tungsten wires housed in a 28 gauge steel cannula beyond which they extended $\sim 1 \mathrm{~mm}$. The wires were cut to yield impedance between 100 and $250 \mathrm{k} \Omega$ at $1 \mathrm{kHz}$. The electrode assembly was fixed to the skull with dental acrylic.

Behavioral apparatus. Fear conditioning was performed in standard rodent conditioning chambers (context "A"; for details, see Maren, 2000). These chambers rested on a load-cell platform that was used to record chamber displacement in response to each rat's motor activity. Load-cell amplifier output from each chamber was digitized at $5 \mathrm{~Hz}$ and acquired online using Threshold Activity software (Med Associates, Burlington, VT). Extinction training and testing took place in two unique contexts ("B" and "C"). These sessions were conducted in the same room, which was separate from the fear conditioning room, in standard rodent conditioning chambers modified to accommodate electrophysiological recording. During these sessions, the load-cell amplifier output was acquired online using DataWave software (DataWave Technologies, Longmont, CO). The three contexts were differentiated through manipulation of multiple visual, olfactory, and tactile cues. Electrophysiological recordings were only made during test sessions in contexts B and C after extinction training. They were made via a recording cable containing an eight-channel field-effect transistor head stage that passed highimpedance signals from the implanted electrode to a computer via a commutator. For each channel, signals were acquired in $3 \mathrm{sec}$ epochs (500 msec pre-CS, $2 \mathrm{sec}$ CS, $500 \mathrm{msec}$ post-CS) for 10 test trials. All neuronal signals were amplified (gain of 10,000), filtered $(600-9000 \mathrm{~Hz}$ ), and acquired and digitized using Experimenter's Workbench software (DataWave Technologies).

Procedure. All experimental animals [extinction (EXT); $n=7$ ] were fear conditioned in context A by administering 10 tone $(2 \mathrm{kHz}, 80 \mathrm{~dB}, 2$ sec)-shock ( $1 \mathrm{~mA}, 0.5 \mathrm{sec})$ pairings and 10 white noise $(80 \mathrm{~dB}, 2 \mathrm{sec})-$ shock $(1 \mathrm{~mA}, 0.5 \mathrm{sec})$ pairings. Tone and white noise trials were alternated [62 sec intertrial interval (ITI)]. The following day, rats were extinguished to one CS in context B and the other CS in context C. Some rats were extinguished to the white noise in $\mathrm{B}$ and the tone in $\mathrm{C}$, whereas other rats received the opposite cue-context combination. During each extinction session, rats received $30 \mathrm{CS}$ presentations after placement into the chamber (62 sec ITI). Extinction sessions for each CS were performed once per day for $3 \mathrm{~d}$, yielding a total of 90 extinction trials per CS.

The day after the last extinction session, all animals were implanted with a recording electrode aimed at the LAd. After recovery, fear memory retrieval was tested by presenting each CS in each extinction context, yielding a total of four retrieval tests. Hence, each CS was tested in its own extinction context [consistent condition $(\mathrm{CON})]$ or another extinction context [inconsistent condition (INCON)]. Test sessions consisted of 10 CS presentations beginning $\sim 1$ min after attaching the recording cable and placing the animal in the test context ( $62 \mathrm{sec}$ ITI). Rats were returned to their home cages immediately after testing. Retrieval tests on each day were administered at least $1.5 \mathrm{hr}$ apart. Tests in context $\mathrm{B}$ were conducted on the first test day, and tests in context $\mathrm{C}$ were conducted on the second test day. Rats may have received the INCON or CON test first on each of those days. Importantly, each rat in the EXT group served as its own control; that is, contextual modulation of behavior and neuronal activity were examined within each subject.

Two control groups were included to assess whether contextual modulation of behavior and neuronal activity is specific to extinguished CSs. One group was presented non-extinguished CSs [no extinction (NOEXT); $n=5$ ] in each context, and the other was presented auditory cues that were never paired with shock [habituation $(\mathrm{HAB}) ; n=5$ ]; that is, the $\mathrm{NO}-\mathrm{EXT}$ and the HAB control groups were treated identically to the EXT group with the following exceptions: NO-EXT rats were not presented with CSs in the extinction contexts, whereas $\mathrm{HAB}$ rats were not presented with USs during the training phase. Importantly, in the HAB group, each auditory cue was tested in the context in which it had repeatedly been presented $(\mathrm{CON})$ and in the context in which the other cue had been presented (INCON). Conditional freezing, a measure of fear, was recorded during all phases of the experiment, and neuronal responses were recorded during each test session.

Histology. After retrieval testing, the animals were overdosed with sodium pentobarbital, and a small lesion was made at the tip of one of the electrodes by passing anodal current $(80 \mu \mathrm{A}, 10 \mathrm{sec})$. Thereafter, animals were perfused across the heart using physiological saline solution, followed by a $10 \%$ Formalin solution. Brains were removed and placed in $10 \%$ Formalin or in a 10\% Formalin-30\% sucrose solution. Before sectioning, all brains were placed in 10\% Formalin-30\% sucrose for at least $4 \mathrm{hr}$. Using a cryostat, brains were sectioned $(40-45 \mu \mathrm{m})$, mounted on microscope slides, and stained with $0.25 \%$ thionin to verify electrode placements.

Data analysis. Only those animals with electrode placement in the LA or amygdalostriatal transition area were included in the analysis (Fig. 1). Freezing data were transformed into a percentage of total observations, and these percentages were analyzed using ANOVA. Post hoc comparisons were conducted using Tukey's honestly significant difference test. All neuronal analysis was performed offline using Autocut (DataWave Technologies) and Neuroexplorer (Plexon, Dallas, TX) software. Single units were isolated from multiple-unit records on each recording channel using standard spike sorting and clustering methods (Maren, 2000). For each unit, spikes were binned ( 10 or $50 \mathrm{msec}$ ) for each $3 \mathrm{sec}$ CS and summed across the 10 test trials. Our analysis was limited to units with a baseline firing rate (firing rate before the onset of the first CS) below 25 $\mathrm{Hz}$ that demonstrated significant CS responsiveness. A cell was CS responsive if, within the first $100 \mathrm{msec}$ after CS onset, its firing was at least three SDs above baseline (pre-CS) firing in any one of the four retrieval tests.

\section{Results}

\section{Behavior}

Conditional freezing averaged across all four retrieval tests in each group is shown in Figure $2 \mathrm{~A}$. Not surprisingly, NO-EXT rats froze more than EXT and HAB rats. This observation was confirmed by a significant effect of group $\left(F_{(2,14)}=10.8 ; p<0.01\right)$ in the ANOVA. Post hoc comparisons $(p<0.05)$ revealed that freezing in NO-EXT rats was greater than that in EXT and HAB rats, and freezing in EXT rats was greater than that in HAB rats during interstimulus intervals two and three. Importantly, conditional freezing in the EXT group was contextually modulated; EXT rats froze less to a CS when it was presented in its extinction context $(\mathrm{CON})$ than to a CS presented outside of its extinction context (INCON). Figure $2 B$ shows the ratio of freezing during the INCON and CON retrieval tests in EXT and HAB rats [modulation ratio $=(\mathrm{INCON}$ freezing $) /(\mathrm{CON}$ freezing $)]$. For this ratio, a value equal to one indicates an absence of contextual modulation (equal levels of freezing during the CON and INCON tests). One-sample $t$ tests confirmed that the modulation ratio in EXT rats was significantly $>1\left(t_{(6)}=2.1 ; p<0.05\right)$; the ratio was not significantly different from 1 in $\mathrm{HAB}$ rats $\left(t_{(4)}=-1.7 ; p=\right.$ 0.08). Hence, EXT rats exhibited more fear to a CS in the INCON condition compared with the CON condition than did HAB rats. 


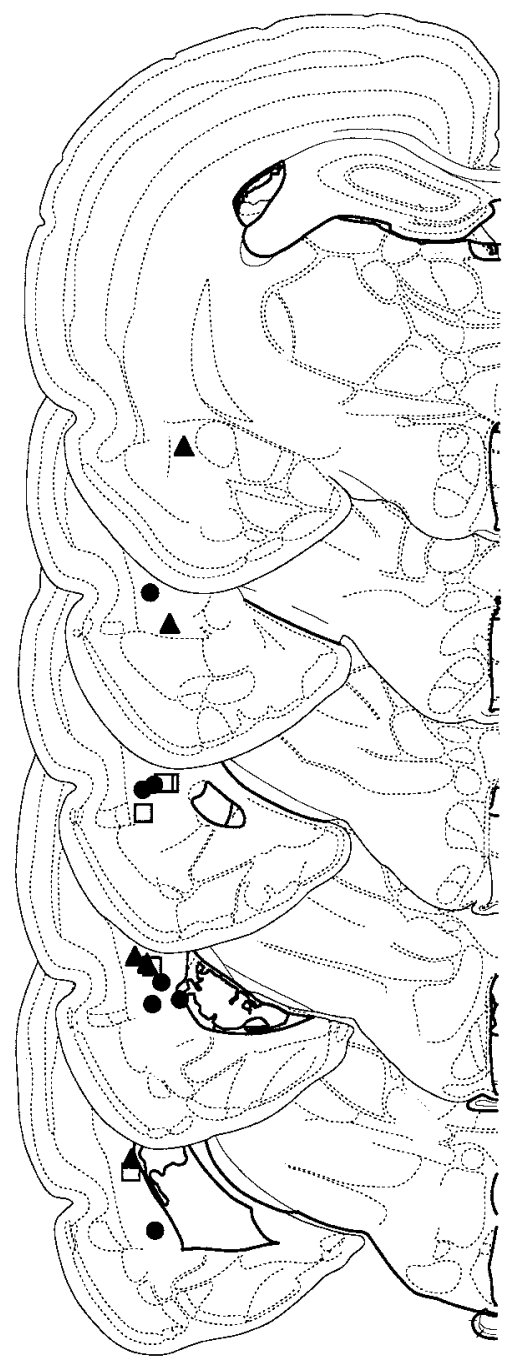

Figure 1. Recording electrode placements in lateral amygdala. Placements represent all NO-EXT (filled triangles), HAB (open squares), and EXT (filled circles) rats included in the final analysis. Schematic brain section images are displayed from most rostral (top; $-2.0 \mathrm{~mm}$ posterior to bregma) to most caudal (bottom; $-3.9 \mathrm{~mm}$ posterior to bregma). Images were adapted from Swanson (1992).

Hence, contextual cues modulated freezing behavior after extinction (Corcoran and Maren, 2001).

\section{Spike firing}

To ascertain the neural basis for context-dependent fear memory, we recorded single-unit activity in the LA during each retrieval test using a multi-channel electrode assembly. One hundred nineteen of $151(78.8 \%)$ LA units we recorded were CS responsive during retrieval testing (EXT, 59 of 76; NO-EXT, 41 of 51; $\mathrm{HAB}, 19$ of 24). To examine the baseline firing rates of these neurons, we examined the average neuronal firing during the 0.5 sec period before the onset of the first CS of each test session. Fifteen neurons were excluded from this analysis because of the presence of noise that precluded spike detection during this time period. The mean \pm SEM baseline firing rates of the EXT, NO-EXT, and HAB groups were $2.6 \pm 0.5,5.1 \pm 0.6$, and $7.4 \pm 0.9 \mathrm{~Hz}$, respectively. An ANOVA performed on these means showed a significant effect of group $\left(F_{(2,101)}=15.7 ; p<0.00001\right)$. Post hoc $(p<0.05)$ comparisons revealed that the units recorded from $\mathrm{HAB}$ and NO-EXT rats fired more than those recorded from EXT rats.
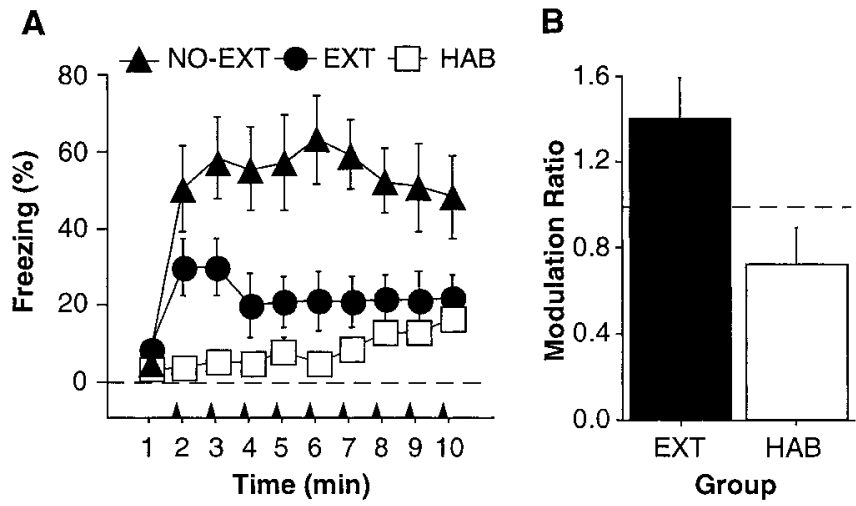

Figure 2. Context-specific expression of conditional freezing. $A$, Mean \pm SEM percentage of freezing during the 1 min interstimulus intervals averaged across all retrieval tests for NO-EXT (filled triangles), EXT (filled circles), and HAB (open squares) rats. The arrowheads indicate the onset of the CSS. $B$, Ratio of the average (mean \pm SEM) percentage of freezing during the INCON tests and the $\mathrm{CON}$ tests. A value of 1 (dashed line) indicates equivalent freezing during the $\mathrm{CON}$ and INCON tests.

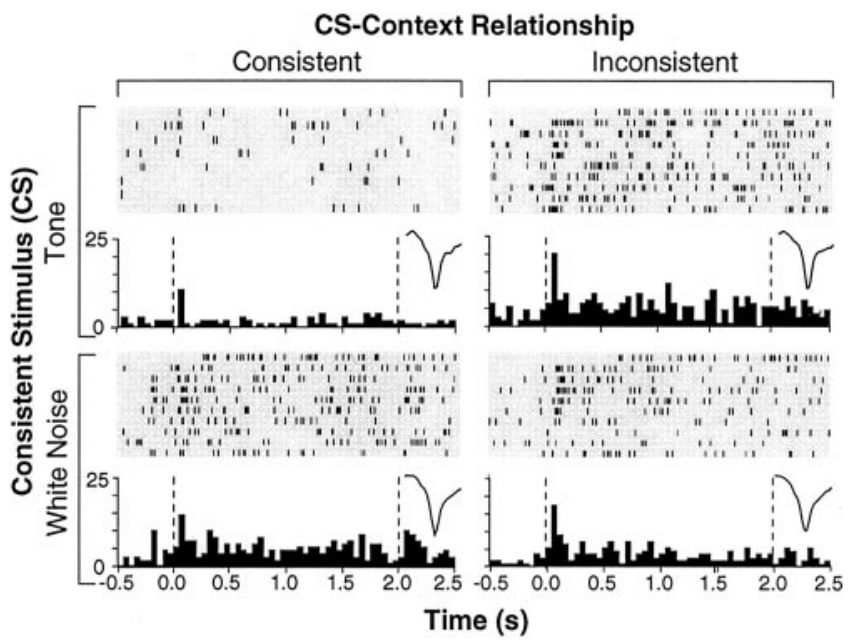

Figure 3. Raster plots and histograms derived from a representative LA unit from the EXT group. This neuron (average spike waveform for each test shown in the insets) exhibited greater CS-evoked spike firing during INCON compared with CON tests. Dashed lines in the histograms indicate the onset and offset of the $2 \mathrm{sec}$ CS. Raster plots show spikes detected during the $3 \mathrm{sec}$ sweep collected on each of the 10 CS trials delivered during each retrieval test. Peristimulus time histograms represent the sum of these spikes across the 10 trials (binned in 50 msec intervals).

Importantly, we found that neurons recorded from rats in the EXT group exhibited contextually modulated spike firing; that is, the neuronal responses to extinguished auditory CSs were modulated by the relationship between the CS and the test context. For example, the single unit shown in Figure 3 exhibited greater CS-evoked firing shortly after CS onset when the auditory CSs were presented in the INCON condition. This contextdependent firing was not attributable to any intrinsic property of the contexts themselves or to the reinforcement history of the contexts; both test contexts had hosted extinction training to one of the CSs.

To further characterize CS-elicited activity in the population of neurons we recorded in each group, we normalized the CSelicited spike firing for each cell to its pre-CS firing rate. Peristimulus histograms depicting the normalized neuronal activity averaged across all cells in each group are shown in Figure 4. As shown, the overall CS-evoked activity differed among groups. An ANOVA performed on the average overall CS-evoked activity 


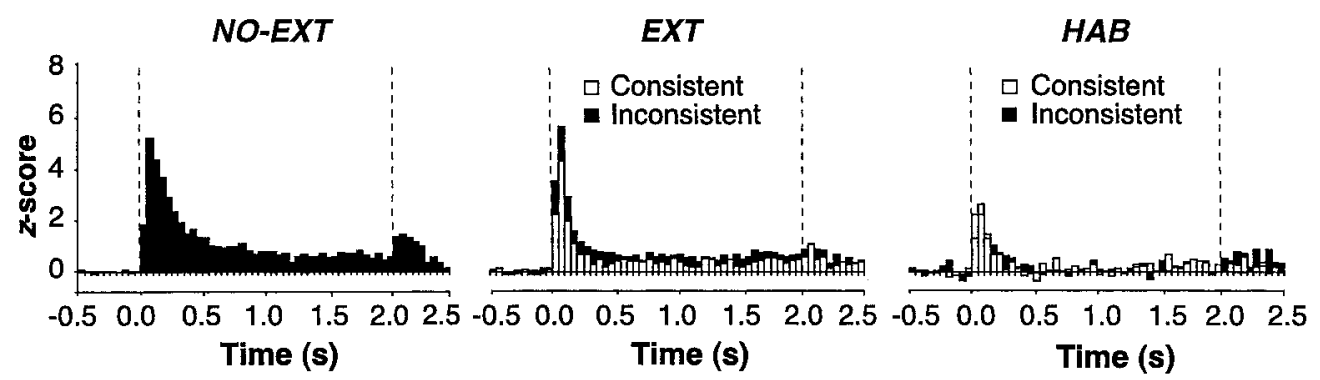

Figure 4. Normalized neuronal activity for all units in each group. Spike firing was summed across the $10 \mathrm{CS}$ trials in each retrieval test, and post-CS activity was normalized to the pre-CS baseline $(50 \mathrm{msec}$ bins; a total of $500 \mathrm{msec}$ before CS onset). Standard scores (z-scores) for each unit were averaged across all of the retrieval tests in the N0-EXT rats (filled bars) and were averaged across the CON (open bars) and INCON (filled bars) tests in the EXT and HAB groups. Dashed lines indicate CS onset and offset.

A

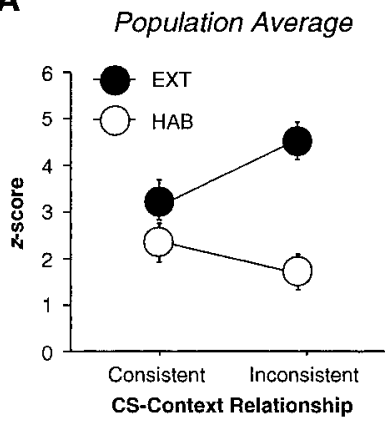

B

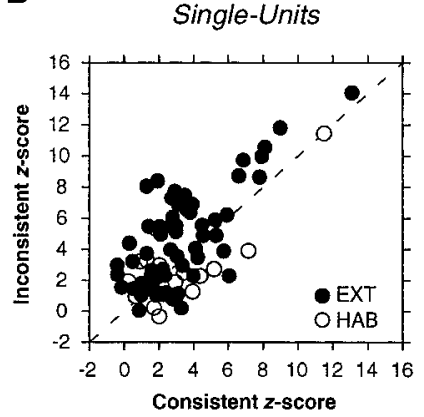

Figure 5. $\quad A$, Mean \pm SEM short-latency $(0-100 \mathrm{msec})$ CS-evoked single-unit activity for the EXT (filled circles) and HAB (open circles) rats. $B$, Scatter plot showing the normalized post-CS onset $(0-100$ msec) firing rate for INCON ( $y$-axis) and CON ( $x$-axis) tests for every neuron recorded in the EXT (filled circles) and $\mathrm{HAB}$ (open circles) groups. Dashed line indicates a $45^{\circ}$ line.

confirmed that there was a main effect of group $\left(F_{(2,116)}=17.9\right.$; $p<0.00001)$. Post hoc comparisons $(p<0.05)$ revealed that $\mathrm{CS}$-evoked firing was greatest in the NO-EXT rats, intermediate in the EXT rats, and lowest in the HAB rats. As reported previously (Quirk et al., 1995, 1997; Maren, 2000; Paré and Collins, 2000), CS-elicited firing was maximal within the first $100 \mathrm{msec}$ of CS onset and was present within $20 \mathrm{msec}$ of CS onset (data not shown). However, CS-evoked neuronal activity in the EXT group was context dependent in that the same auditory CSs evoked different levels of spike firing depending on the context in which they were presented. In general, LA units exhibited greater levels of firing to extinguished CSs when they were presented outside of their extinction context (INCON condition).

These observations were confirmed by an analysis of CSevoked neuronal activity during the first $100 \mathrm{msec}$ after CS onset. As shown in Figure $5 A$, short-latency CS-evoked activity was greater during INCON tests than during CON tests for animals in the EXT group. An ANOVA performed on these averages revealed a main effect of group $\left(F_{(2,76)}=8.5 ; p<0.01\right)$ and a group by test-type interaction $\left(F_{(2,76)}=13.7 ; p<0.001\right)$. Post hoc comparisons $(p<0.05)$ revealed greater firing during INCON tests compared with CON tests in the EXT group. Importantly, there were no within-group differences in the $\mathrm{HAB}$ group, indicating that spike firing in this group was not contextually modulated. Additional analysis revealed that modulation of LA activity appeared as early as $40-50 \mathrm{msec}$ after CS onset. CS-evoked activity during the first $100 \mathrm{msec}$ after CS onset was examined in $10 \mathrm{msec}$ bins. An ANOVA performed on these data revealed a main effect of test type $\left(F_{(1,58)}=9.7 ; p<0.01\right)$ and a main effect of bin $\left(F_{(9,522)}=19.8 ; p<0.0001\right)$. Post hoc comparisons $(p<0.05)$

revealed greater spike firing to INCON compared with $\mathrm{CON}$ in the $40-50$ and $60-70$ msec bins.

The population averages suggest that the majority of neurons in the EXT group exhibited greater CS-evoked spike firing during INCON compared with CON tests. To examine this at the level of single neurons, short-latency CS-evoked firing (0-100 msec after CS-onset) during the INCON and CON tests was plotted against one another for the EXT and HAB groups (Fig. $5 B$ ). The majority of neurons $(74.6 \%)$ in the EXT group fired more during the INCON compared with the CON test (these points fall above the $45^{\circ}$ line on the scatter plot). In contrast, slightly more than onehalf of the HAB neurons (63.2\%) fired more during the CON test. Nonetheless, the average magnitude of CS-elicited spike firing was only context dependent in the EXT animals (Fig. 5A).

Importantly, although it is not clear what underlies the differences in baseline firing rate observed among the three groups, these differences do not appear to be responsible for the contextdependent firing we observed. An ANOVA performed on HAB units with baseline firing rates within $1 \mathrm{SD}$ of the mean firing rate of EXT neurons failed to reveal a difference between CON and INCON test sessions $\left(F_{(1,4)}=1.79 ; p>0.05\right)$. Additionally, an ANOVA performed on EXT units with baseline firing rates within $1 \mathrm{SD}$ of the mean firing rate of $\mathrm{HAB}$ units still showed greater firing during INCON compared with CON sessions $\left(F_{(1,13)}=9.71 ; p<0.01\right)$.

\section{Discussion}

The results of the present study demonstrate that acoustic CSs with the same reinforcement histories are represented in the LA by different neural codes depending on the context in which they are presented. Importantly, the frequency of spike firing in the LA was greater when CSs were presented outside compared with inside the extinction context. Moreover, this contextual modulation was only evident for stimuli that had an ambiguous meaning (i.e., extinguished CSs) and not stimuli that unambiguously predicted shock (i.e., non-extinguished CSs) or stimuli that never predicted shock (i.e., cues never paired with shock). These data suggest that LA neurons represent the meaning of ambiguous CSs by a context-dependent rate code, and we propose that this neural representation, in turn, generates the context-specific expression of conditional fear responses.

It is possible, however, that the activity of LA neurons does not cause context-specific behavior but rather results from contextspecific behavior to the CS. For example, the freezing posture may alter sensory transduction of the auditory CS (by altered position of the pinnae, for example) or fear arousal may nonspecifically alter neuronal processing of CS information in the LAd. Consistent with this view, CS-elicited spike firing is almost always 

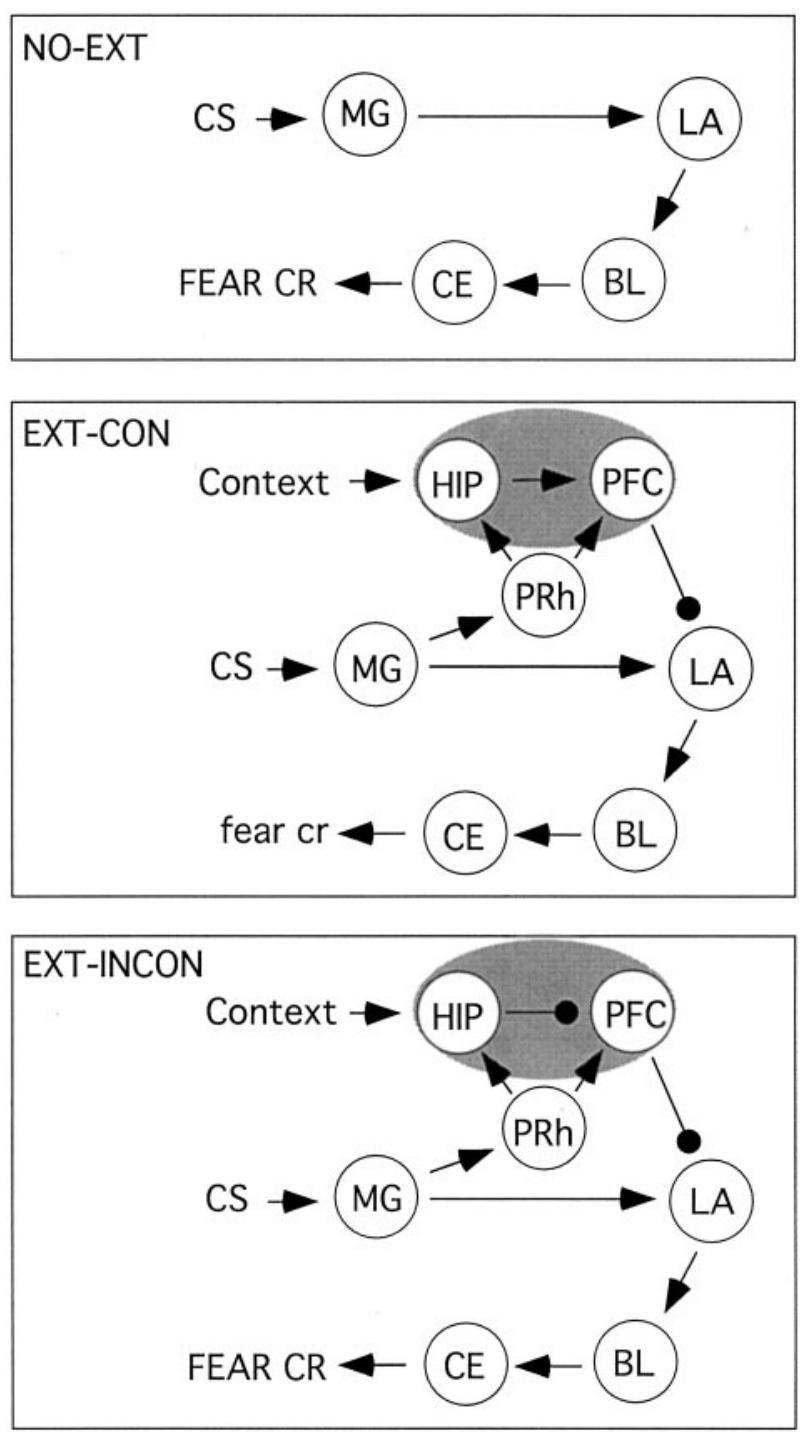

Figure 6. A model of contextual modulation of CS-elicited spike firing and conditional freezing after Pavlovian fear conditioning. In non-extinguished rats, auditory fear conditioning is not context specific (NO-EXT; top). In this case, auditory CSs are transmitted to the LA via the medial geniculate nucleus of the thalamus (MG). Excitatory inputs are then sent to the basolateral amygdala (BL) and the central nucleus ( $(\mathrm{E})$, which generates the expression of $(\mathrm{Rs}$. We propose that, during extinction, the medial geniculate nucleus of the thalamus transmits $C S$ information to the hippocampus (HIP) and the prefrontal cortex (PFC) via cortical structures such as the perirhinal (PRh), entorhinal, or primary auditory cortices. The prefrontal cortex in turn sends inhibitory inputs to LA that dampen CR expression. This can occur in the absence of hippocampus input to the prefrontal cortex. During extinction training, however, the hippocampus encodes a conjunctive representation of the $\mathrm{CS}$ - context relationship and transmits this information to the prefrontal cortex. When animals are tested in the extinction context (EXT-CON; middle panel), prefrontal coretx inhibition of LA prevails. In an extinction-inconsistent context (EXT-INCON; bottom), however, the CS-context conjunction is not activated and the hippocampus sends inhibitory inputs to the prefrontal cortex. The expression of the $C R$ is not inhibited under these conditions.

positively correlated with conditional freezing (Quirk et al., 1995, 1997; Maren, 2000). In the present study, spike firing was greatest in the INCON sessions when the animals were freezing the most and lowest in the CON sessions when they were freezing the least. However, a recent report from our laboratory suggests that freezing behavior is neither necessary nor sufficient to increase CSelicited spike firing in LAd (Goosens et al., 2002). We trained animals in a discriminative fear conditioning procedure that al- lowed us to examine neuronal responses to CSs with different reinforcement histories under conditions in which freezing to both CSs was equally high. We found more robust neuronal discrimination to stimuli that had been paired with shock compared with stimuli that had not, although freezing to the two cues was the same. Additionally, we demonstrated that preventing conditional freezing by pharmacological inactivation of the central nucleus of the amygdala (CE) before extinction testing did not prevent the development of conditional unit activity in LA. These data suggest that CS-elicited spike firing in LA reflects the associative coding of auditory CSs and is not simply a neural representation of conditional freezing.

Together, the data described above suggest that CS-elicited spike firing in the LA after extinction represents the CS-context relationship. There is consensus that conditioning and extinction lead to the formation of two separate representations of the CS (for review, see Bouton, 1993). The conditioning memory, or CS-US memory, is excitatory, whereas the competing extinction (CS-no US) memory is inhibitory. It has been proposed that the extinction context gates activation of the inhibitory memory and consequent suppression of fear responses in the extinction context. In all other contexts, the presentation of the CS activates memory for the CS-US association leading to the expression of fear responses (Bouton, 1993; Maren and Holt, 2000). The data reported here are consistent with the existence of dual representations of the CS after extinction. In our experiment, a single CS was able to excite or inhibit fear responses depending on the context in which that CS was presented. Although numerous studies demonstrate that the basolateral amygdala (BLA) is involved in the acquisition, storage, and retrieval of the excitatory CS-US memory (Davis, 1992; LeDoux, 2000; Maren, 2001), less is known about the coding of the extinction memory.

Recent data suggest a role for NMDA receptor-dependent mechanisms in the BLA in establishing the extinction memory. Intra-BLA application of NMDA receptor antagonists (Falls et al., 1992) and mitogen-activated protein kinase inhibitors impair fear memory extinction (Lu et al., 2001), whereas NMDA receptor agonists enhance extinction (Walker et al., 2002). Additionally, it is likely that GABAergic transmission is involved in fear memory extinction. Systemic administration of the $\mathrm{GABA}_{\mathrm{A}}$ antagonist picrotoxin after the extinction of inhibitory avoidance learning enhanced extinction retention during testing (McGaugh et al., 1990), whereas the $\mathrm{GABA}_{\mathrm{A}}$-positive allosteric modulator diazepam impaired extinction retention when administered before extinction in a shuttle avoidance task (Pereira et al., 1989). Royer and Paré (2002) suggest that intercalated amygdala (IC) may be a locus of fear memory inhibition. These GABAergic neurons are optimally located to gate the excitatory inputs of the BLA to the CE, enabling the suppression of CE activity and CRs. Although it is possible that the locus or modulation of the extinction memory is downstream of the LA, this does not explain the contextual modulation of LA neurons reported here, the locus of which is upstream of IC. However, recent evidence suggests that an inhibitory network capable of gating LA responses exists locally within the LA (Shumyatsky et al., 2002). Gastrin-related peptide (GRP) has been shown to excite GABAergic interneurons in LA, leading to the enhanced inhibition of principal amygdala neurons. Moreover, GRP receptor knock-out mice show enhanced LTP in the fear circuit and greater and more persistent conditional fear responses. Finally, work by Harris and Westbrook (1998) demonstrates that administration of the $\mathrm{GABA}_{\mathrm{A}}$ inverse agonist $\beta$-carboline attenuates both the acquisition and expression of fear memory extinction. Importantly, $\beta$-carboline 
only disrupted the expression of extinction in the context in which extinction training took place. This outcome would be predicted if the activation of GABAergic interneurons in LA mediates the activation of the CS-no-US association but not the CS-US association. To further examine the role of GABAergic interneurons in extinction, future studies will ideally use recording techniques that enable interneuron firing to be discriminated from principal neuron firing.

If both conditioning and extinction memories reside in the amygdala, it seems reasonable that modulation of the expression of those memories occurs there as well. However, it is possible that the context-specific CS-elicited spike firing we observed originates afferent to the amygdala. Previous research demonstrates that projections from the medial geniculate nucleus of the auditory thalamus to the LA are involved in CS processing during auditory fear conditioning (Romanski and LeDoux, 1992; Quirk et al., 1995, 1997). However, single-unit recording in LA demonstrates that the latency of tone-evoked responses in the thalamoamygdala pathway can occur $<20 \mathrm{msec}$ after CS onset. Toneevoked responses with latencies $>20 \mathrm{msec}$, on the other hand, may reflect transmission through the thalamo-cortico-amygdala or thalamo-amygdala pathways (Quirk et al., 1997). In the present experiment, contextual modulation of LAd responses was not manifest until 40-50 msec after CS onset, suggesting that this modulation occurs downstream of the auditory thalamus.

Indeed, the contextual modulation of amygdaloid spike firing is likely mediated by an interaction of amygdala neurons with other brain circuits that encode contextual information. One brain structure that has a prominent role in processing context is the hippocampus. Several studies have shown that the hippocampus has an important role in encoding contextual representations (Rudy et al., 2002) (for review, see Anagnostaras et al., 2001; Sanders et al., 2003). Moreover, several authors have also suggested that the hippocampus is essential for using contextual information to label and retrieve memories (Hirsh, 1974; Good and Honey, 1991; Maren and Holt, 2000; Rudy and O'Reilly, 2001). Consistent with this view, research from our laboratory suggests that the neural circuit mediating the context dependency of amygdala spike firing may involve the hippocampus (Fig. 6). We showed that reversible inactivation of the dorsal hippocampus with the GABAA agonist muscimol impairs the context-specific expression of both latent inhibition (Holt and Maren, 1999) and extinction (Corcoran and Maren, 2001). These data suggest that information about the relationship between the CS and the context in which it is extinguished may be encoded by the hippocampus. This information may regulate the expression of excitatory and inhibitory fear memories via direct or indirect hippocampoamygdala interactions.

In addition to the amygdala and hippocampus, the prefrontal cortex may also play a role in the representation of extinction memories (Fig. 6). Animals with lesions of the infralimbic cortex show within-session extinction learning but do not maintain extinction-level responding when tested the following day (Quirk et al., 2000). These data, as well as electrophysiological data showing that electrical stimulation of infralimbic cortex simulates extinction memory (Milad and Quirk, 2002), suggest that infralimbic cortex may be a locus of consolidation or storage of extinction memories. These data also leave open the possibility that infralimbic cortex is involved in representing contextual information that enables the appropriate activation of the extinction memory (Quirk et al., 2000). It will be of interest to determine whether this structure interacts with the hippocampus and amygdala to consolidate or otherwise represent the conjunction of contextual in- formation and the inhibitory CS associations formed during extinction. Finally, interactions between the entorhinal cortex and the amygdala appear to be essential for contextual modulation in a discriminative avoidance task (Freeman et al., 1997), and it will be of interest to determine whether this circuitry is necessary for contextual modulation of Pavlovian fear memory extinction.

Context-dependent spike firing in the LA during fear memory retrieval may have important implications for understanding clinical fear and anxiety disorders. These disorders are characterized by the inability to inhibit fear responses in nonthreatening or otherwise inappropriate situations. Current conditioning-based explanations of these disorders point to the influence of contextual cues in triggering their expression after extinction (Bouton et al., 2001). A substantial body of research indicates that GABA transmission may mediate these disorders. Indeed, the administration of benzodiazepines has been widely used in the clinical treatment of anxiety (Gorman, 2003), and extensive animal research points to the anxiety-mediating effects of increased and decreased GABA transmission (Sanders and Shekhar, 1995; de Jongh et al., 2002; Kalin, 2003). Understanding the neural and behavioral mechanisms by which contextual information regulates the expression of fear may enable the development of treatments for these disorders. Ideally, treatment would allow patients to minimize the context-inappropriate expression of fear responses while maintaining the ability to express contextappropriate fear.

\section{References}

Albright TD, Stoner GR (2002) Contextual influences on visual processing. Annu Rev Neurosci 25:339-379.

Anagnostaras SG, Gale GD, Fanselow MS (2001) Hippocampus and contextual fear conditioning: recent controversies and advances. Hippocampus $11: 8-17$.

Bouton ME (1993) Context, time, and memory retrieval in the interference paradigms of Pavlovian learning. Psychol Bull 114:80-99.

Bouton ME, Bolles RC (1979) Contextual control of the extinction of conditioned fear. Learn Motiv 10:445-466.

Bouton ME, Mineka S, Barlow DH (2001) A modern learning theory perspective on the etiology of panic disorder. Psychol Rev 108:4-32.

Corcoran KA, Maren S (2001) Hippocampal inactivation disrupts contextual retrieval of fear memory after extinction. J Neurosci 21:1720-1726.

Das A, Gilbert CD (1999) Topography of contextual modulations mediated by short-range interactions in primary visual cortex. Nature 399:655-661

Davis M (1992) The role of the amygdala in fear and anxiety. Annu Rev Neurosci 15:353-375.

de Jongh R, Groenink L, van Der Gugten J, Olivier B (2002) The lightenhanced startle paradigm as a putative animal model for anxiety: effects of chlordiazepoxide, flesinoxan and fluvoxamine. Psychopharmacology 159:176-180.

Diamond DM, Weinberger NM (1989) Role of context in the expression of learning-induced plasticity of single neurons in auditory cortex. Behav Neurosci 103:471-494.

Falls WA, Miserendino MJ, Davis M (1992) Extinction of fear-potentiated startle: blockade by infusion of an NMDA antagonist into the amygdala. J Neurosci 12:854-863.

Freeman JH, Wieble A, Rossi J, Gabriel M (1997) Lesions of the entorhinal cortex disrupt behavioral and neural responses to context change during extinction of discriminative avoidance behavior. Exp Brain Res 115:445-457.

Good MC, Honey RC (1991) Conditioning and contextual retrieval in hippocampal rats. Behav Neurosci 105:499-509.

Goosens KA, Hobin JA, Maren S (2002) Conditional increases in amygdala unit activity are regulated by associative factors and not the expression of fear. Soc Neurosci Abstr 28:83.17.

Gorman JM (2003) New molecular targets for antianxiety interventions. J Clin Psychiatry 64:28-35.

Harris JA, Westbrook RF (1998) Evidence that GABA transmission medi- 
ates the context-specific extinction of learned fear. Psychopharmacology 140:105-115.

Harris JA, Jones ML, Bailey GK, Westbrook RF (2000) Contextual control over conditioned responding in an extinction paradigm. J Exp Psychol Anim Behav Process 26:174-185.

Herzog MH, Fahle M (2002) Effects of grouping in contextual modulation. Nature 415:433-436.

Hirsh R (1974) The hippocampus and contextual retrieval of information from memory: a theory. Behav Biol 12:421-444.

Holt W, Maren S (1999) Muscimol inactivation of the dorsal hippocampus impairs contextual retrieval of fear memory. J Neurosci 19:9054-9062.

Kalin NH (2003) Nonhuman primate studies of fear, anxiety, and temperament and the role of benzodiazepine receptors and GABA systems. J Clin Psychiatry 264:41-44.

LeDoux JE (2000) Emotion circuits in the brain. Annu Rev Neurosci 23:155-184.

Lu KT, Walker DL, Davis M (2001) Mitogen-activated protein kinase cascade in the basolateral amygdala is involved in extinction of fearpotentiated startle. J Neurosci 21:RC162(1-5).

Malone BJ, Scott BH, Semple MN (2002) Context dependent adaptive coding of interaural phase disparity in the auditory cortex of awake macaques. J Neurosci 22:4625-4632.

Maren S (2000) Auditory fear conditioning increases CS-elicited spike firing in lateral amygdala neurons even after extensive overtraining. Eur J Neurosci 12:4047-4054.

Maren S (2001) Neurobiology of Pavlovian fear conditioning. Annu Rev Neurosci 24:897-931.

Maren S, Holt WG (2000) The hippocampus and contextual memory retrieval in Pavlovian conditioning. Behav Brain Res 110:97-108.

McGaugh JL, Castellano C, Brioni J (1990) Picrotoxin enhances latent extinction of conditioned fear. Behav Neurosci 104:264-267.

Milad MR, Quirk GJ (2002) Neurons in medial prefrontal cortex signal memory for fear extinction. Nature 420:70-74.

Paré D, Collins DR (2000) Differential fear conditioning induces reciprocal changes in the sensory responses of lateral amygdala neurons to the CS $(+)$ and CS (-). J Neurosci 20:2701-2710.

Pereira ME, Rosat R, Huang CH, Godoy MG, Izquierdo I (1989) Inhibition by diazepam of the effect of additional training and of extinction on the retention of shuttle avoidance behavior in rats. Behav Neurosci 103:202-205.
Quirk GJ, Repa C, LeDoux JE (1995) Fear conditioning enhances shortlatency auditory responses of lateral amygdala neurons: parallel recordings in the freely behaving rat. Neuron 15:1029-1039.

Quirk GJ, Armony JL, LeDoux JE (1997) Fear conditioning enhances different temporal components of tone-evoked spike trains in auditory cortex and lateral amygdala. Neuron 19:613-624.

Quirk GJ, Russo GK, Barron JL, Lebron K (2000) The role of ventromedial prefrontal cortex in the recovery of extinguished fear. J Neurosci 20:6225-6231.

Romanski LM, LeDoux JE (1992) Equipotentiality of thalamo-amygdala and thalamo-corticoamygdala circuits in auditory fear conditioning. J Neurosci 12:4501-4509.

Rossi AF, Desimone R, Ungerleider LG (2001) Contextual modulation in primary visual cortex of macaques. J Neurosci 21:1698-709.

Royer S, Paré D (2002) Bidirectional synaptic plasticity in intercalated amygdala neurons and the extinction of conditioned fear responses. Neuroscience 115:455-462.

Rudy JW, O’Reilly RC (2001) Conjunctive representations, the hippocampus, and contextual fear conditioning. Cogn Affect Behav Neurosci $1: 66-82$.

Rudy JW, Barrientos RM, O'Reilly RC (2002) Hippocampal formation supports conditioning to memory of a context. Behav Neurosci 116:530-538.

Sanders SK, Shekhar A (1995) Regulation of anxiety by $\mathrm{GABA}_{\mathrm{A}}$ receptors in the rat amygdala. Pharmacol Biochem Behav 52:701-706.

Sanders MJ, Wiltgen BJ, Fanselow MS (2003) The place of the hippocampus in fear conditioning. Eur J Pharmacol 463:217-223.

Shumyatsky GP, Tsvetkov E, Malleret G, Vronskaya S, Hatton M, Hampton L, Battey JF, Dulac C, Kandel ER, Bolshakov VY (2002) Identification of a signaling network in lateral nucleus of amygdala important for inhibiting memory specifically related to learned fear. Cell 111:905-918.

Swanson LW (1992) Brain maps: structure of the rat brain. New York: Elsevier.

Walker DL, Ressler KJ, Lu KT, Davis M (2002) Facilitation of conditioned fear extinction by systemic administration or intra-amygdala infusions of D-cycloserine as assessed with fear-potentiated startle in rats. J Neurosci 22:2343-2351.

Zipser K, Lamme VA, Schiller PH (1996) Contextual modulation in primary visual cortex. J Neurosci 16:7376-7389. 